\title{
Fluctuations of doublet splittings using the annular billiard
}

\author{
R. Egydio de Carvalho* and A. P. Mijolaro ${ }^{\dagger}$ \\ Instituto de Geociências e Ciências Exatas, Universidade Estadual Paulista-UNESP 13500-230 Rio Claro, SP, Brazil
}

(Received 20 May 2004; published 18 November 2004)

\begin{abstract}
We study the statistical distribution of quantum energy splittings due to a dynamical tunneling. The system, the annular billiard, has whispering quasimodes due to a discrete symmetry that exists even when chaos is present in the underlying classical dynamics. Symmetric and antisymmetric combinations of these quasimodes correspond to quantum doublet states whose degeneracies decrease as the circles become more eccentric. We construct numerical ensembles composed of splittings for two distinct regimes, one which we call semiclassical for high quantum numbers and high energies where the whispering regions are connected by chaos, and other which we call quantal for low quantum numbers, low energies, and near integrable where dynamical tunneling is not a dominant mechanism. In both cases we observe a variation on the fluctuation amplitudes, but their mean behaviors follow the formula of Leyvraz and Ullmo [J. Phys. A 29, 2529 (1996)]. A description of a three-level collision involving a doublet and a singlet is also provided through a numerical example.
\end{abstract}

DOI: 10.1103/PhysRevE.70.056212 PACS number(s): 05.45.Mt, 05.45.Pq, 05.60.Gg, 03.65.Ge

\section{INTRODUCTION}

Tunneling, when associated with chaotic classical dynamics, has been responsible for appreciable manifestations in many quantum scales, and for this reason it has been the object of many studies. Originally, Davis and Heller [1] introduced the nomenclature of dynamical tunneling to explain forbidden classical motions in systems with some discrete symmetry when no conventional potential barrier is present. However, this kind of tunneling is quite wide because it may occur even in an integrable system, for instance, when a particle jumps from one rotation torus to another one with opposite sense around the hyperbolic fixed point of a separatrix $[2,3]$. One notable work relating classical chaos and tunneling was published by Lin and Ballentine [4] using the double well potential with a periodically time-dependent monochromatic driving force, where the concept of tunneling doublets was introduced. Later, Bohigas et al. [5] defined chaos-assisted tunneling as the increasing of dynamical tunneling effects due to the increasing of chaos on the corresponding classical dynamics, more recently Mouchet and Delande [6] renamed this effect as chaotic tunneling due to the fluctuations observed in the energy splittings. From semiclassical approaches, many authors have developed calculations in order to describe more precisely the effects of dynamical tunneling [7-9]. In Ref. [7], the authors define a quantity called parity of motion, associated with complex solutions of the Hamilton-Jacobi equation, which allows nonclassical motion as dynamical tunneling. In Ref. [8], an approach is presented in which the energy splittings due to tunneling are related to the $S$-matrix elements, which are semiclassically determined by considering complex trajectories. In Ref. [9], the annular billiard is also considered via the scattering theory, wherein the phase splittings are associated with paths connecting the quasimodes, supported by the

\footnotetext{
*Electronic mail: regydio@rc.unesp.br

†Electronic mail: apmas@rc.unesp.br
}

whispering gallery tori, through the chaotic sea.

A splitting distribution generated by ensembles of random matrices [10] was treated by Leyvraz and Ullmo [11] to model the chaotic tunneling process, wherein they have provided analytical expressions to evaluate the probability of occurrence of splittings. Their main assumption is that chaotic tunneling is the only, or at least the dominant, operative mechanism. Associated with this, they pose that it is not necessary that all chaotic states should participate equally in the tunneling process nor that the couplings should be uniform. They have checked their formulas using the coupled quartic oscillator potential for which the numerical and theoretical results have shown a good agreement.

In Ref. [6], the authors considered a one-dimensional time-dependent system, periodic in time and in position, and they have observed that for a particular set of parameters, when the system becomes nearly fully integrable, the statistical distribution of tunneling splittings is also in good agreement with the predictions found in Ref. [11]. Regarding this point, we judged that the annular billiard would be a very good model to analyze the applicability of Leyvraz and Ullmo distribution, firstly due to the our previous knowledge about it [12] and also because this system has a discrete symmetry generating congruent tori, associated with doublet states, where dynamical tunneling is nicely related to the classical dynamics. As is well known, the energy splittings oscillate as a perturbation parameter is changed, so that our proposal in this work is to study these fluctuations in two fashions, one from a statistical point of view constructing ensembles of splittings and other from a local analysis of a three-level interaction.

The article is organized as follows. In Sec. II we briefly present the main points concerning the classical dynamics of the annular billiard, and in Sec. III we show the essential formulas for its quantization. Section IV is devoted to the numerical aspects of the precision of the calculations. In Sec. $\mathrm{V}$ we construct the numerical ensembles and we present the obtained results on the splitting distribution. In Sec. VI we take advantage of this model to obtain a numerical collision between a singlet and a doublet, showing a scenario of col- 


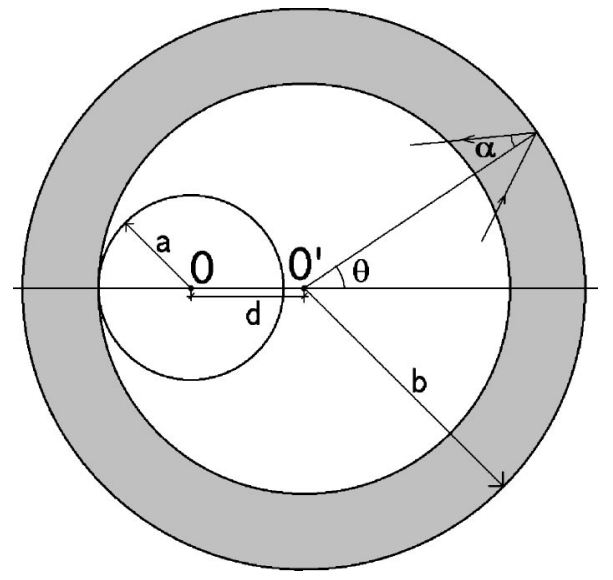
WGO.

FIG. 1. The annular billiard and the region covered by the

lision. Finally, we conclude the article in Sec. VII.

\section{THE MODEL}

The annular billiard $[12,13]$ consists of two circumscribed circles where the inner one may dislocate itself and also change its radius. The outer circle has radius $b$ (in numerical calculations we set $b=1$ ) and the inner circle has radius $a(<b)$. In the accessible ring a particle moves in an uniform rectilinear motion and undergoes elastical reflections on the boundaries. Thus, one has billiard families of two parameters, each family specified by the pair $(a, d)$, where $d$ is the eccentricity. The orbits fall into two classes, the ones which never hit the inner circle, the whispering gallery orbits (WGO), and those which do hit it at least once. Except for the limiting case $a+d=b$, the WGO always exist and they lie inside the shadowed symmetrical annular region of Fig. 1, limited by the outer circle and the caustics, a circle with radius $(a+d)$. The WGO are read in phase space as congruent tori and they are represented there by straight lines. The area of the WGO region remains constant, even if $d$ is varied, when $(a+d)$ is kept constant. This is a very important tool because it ensures that chaos will not "devour" the congruent tori that support the quantum doublets.

The Poincaré sections are described in the variables $L$ $=\theta / 2 \pi$ and $S=\sin (\alpha)$, where $L$ is the normalized arc length and $S$ is the sine of the reflection angle at a collision with the outer circle as presented in Fig. 1. Thus, the ranges of $(L, S)$ are $L:\left[\frac{-1}{2}, \frac{1}{2}\right]$ and $S:[-1,1]$. In Fig. 2, some plots are shown for $(a+d)=0.45$ and for different values of the eccentricity $d$. The chaotic sea grows with $d$ and from $d \gtrsim 0.06$ both borders of WGO, at $|S| \geqslant 0.45$, are connected by chaos.

\section{QUANTIZATION}

The corresponding quantum problem to the annular billiard consists in determining the spectrum of the wave equation

$$
\Delta \Psi+k^{2} \Psi=0
$$

with Dirichlet boundary conditions on the borders, where $k$ is the wavenumber related to the energy $E$ through $E$ $=\hbar^{2} k^{2} / 2 m$. For the concentric case $(d=0)$, besides the energy, the orbital angular momentum is also conserved due to the rotational symmetry. In this case the system is globally integrable and, except for the zero angular momentum states that are singlets, all the others are doublets.

For $d>0$, the angular momentum is no longer conserved. However, the system still has a discrete symmetry under reflections with respect to the line joining the centers of the two circles. Consequently, the eigenstates of Eq. (1) still have well defined parity, but now the boundary condition at the inner circle depends on the angle. Classically, this dependence is read as responsible for introducing chaos to the system and, quantum mechanically, it plays the role of a coupling between states of doublets through chaotic states breaking down their degeneracies $[9,12]$. This mechanism is called dynamical tunneling. One way to solve Eq. (1) is to perform a coordinate transformation through a Fourier expansion, following Refs. [14-16], changing the reference frame to the origin of the inner circle. Thus the even and the odd solutions of the two-dimensional wave equation in polar coordinates $(r, \theta)$ referred to the origin $\mathrm{O}$ (Fig. 1) are

$$
\Psi_{E, O}(r, \theta)=\frac{1}{2} \sum_{i=i 0}^{\infty} \sum_{n=n 0}^{\infty} \in_{i} \in{ }_{n} A_{n} Q_{n i}^{E, O} \times\left\{\begin{array}{l}
\cos i \theta \\
\sin i \theta
\end{array},\right.
$$

where

$$
Q_{n i}^{E, O}=\left[J_{i}(k b)-F_{n}(k a) Y_{i}(k b)\right]\left[J_{i-n}(k d)+(-1)^{n+p} J_{i+n}(k d)\right]
$$

with $n_{0}, i_{0}, p=0$ or $n_{0}, i_{0}, p=1$ for even or odd parity, respectively, and $\epsilon_{j}=2-\delta_{j, 0}$. The quantities $A_{n}$ are integration constants and $F_{n}(k a)=J_{n}(k a) / Y_{n}(k a)$. The eigenwavenumbers $k^{E}$ and $k^{O}$ correspond to the values of $k$ that satisfy

$$
\operatorname{det}\left|Q_{n i}^{E, O}\right|=0 \text {. }
$$

The precision of the numerical results is improved by varying the order of the determinants, and the doublet splittings are given by $\Delta k=\left|k^{E}-k^{O}\right|$. The doublets we consider are built on combinations of WGO tori for which the semiclassical Einstein-Brillouin-Keller (EBK) quantization gives exactly degenerate energies. These tori correspond to horizontal lines lying in the region $|S| \geqslant(a+d)$ in the Poincare section $(L, S)$, whose positions are identified by

$$
\sin \alpha=\frac{n}{k}=S
$$

where $k$ is the unperturbed eigenwavenumber and $S$ is the ordinate of phase space. As we have already pointed out, it is important to consider doublets corresponding to billiard families with $(a+d)$ fixed because as the eccentricity is changed, increasing or decreasing the chaotic sea, the area enclosing the quantized WGO tori remains undisturbed.

\section{NUMERICAL ANALYSIS}

By looking at Eq. (3), we note the Bessel's functions $Z_{n}(\arg )$ should be evaluated in the arguments $k a, k b$, and $k d$, where $b=1$ in all calculations. The range of eigenwavenum- 
a)

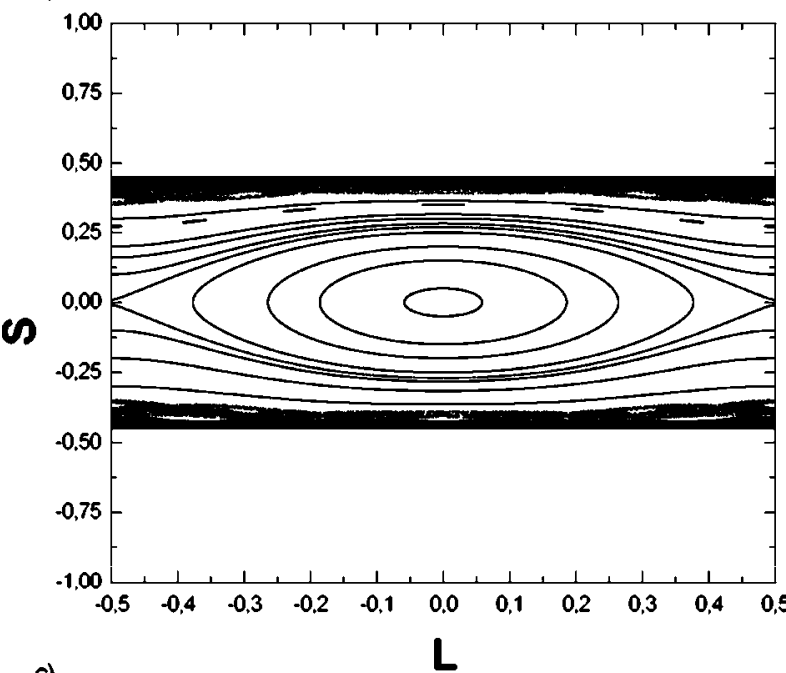

c)

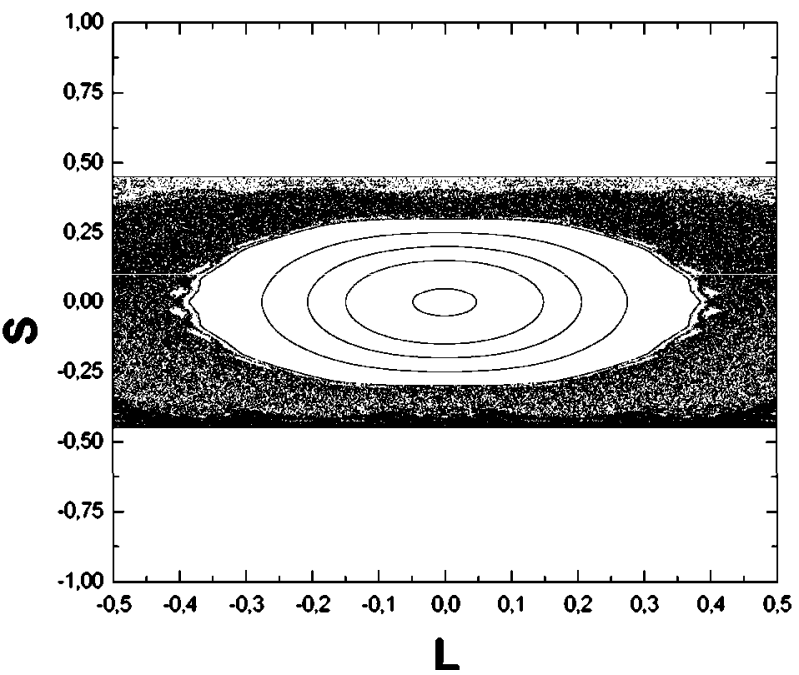

b)

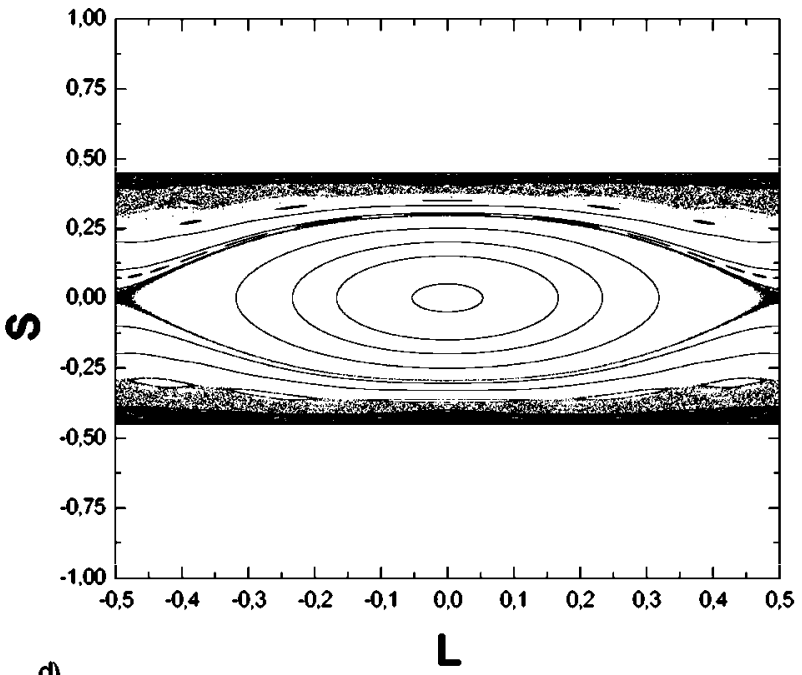

d)

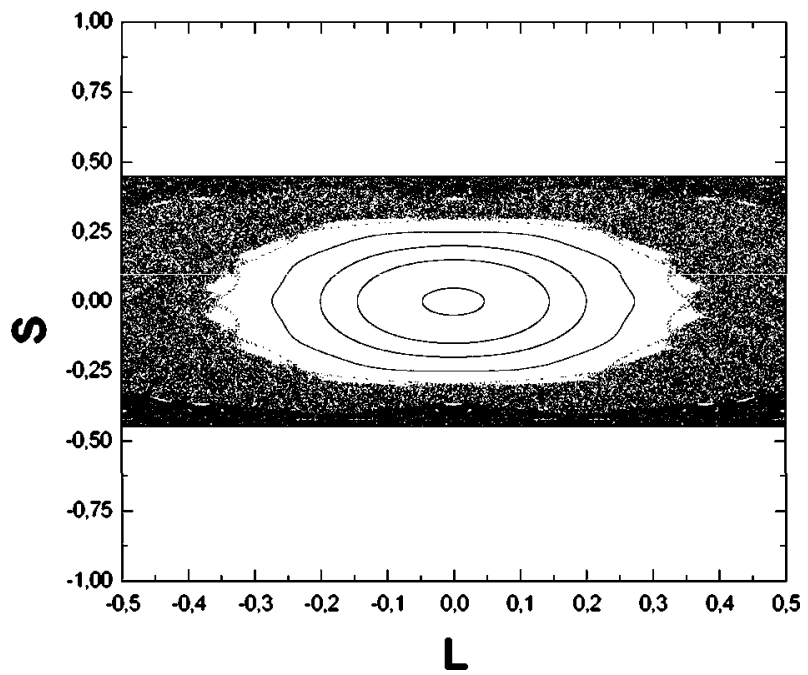

FIG. 2. Poincaré sections with $(a+d)=0.45$ : (a) $d=0.03$, (b) $d=0.04$, (c) $d=0.06$, and (d) $d=0.07$. The units are arbitrary.

bers we have searched was around $k=23-278$, and since the dynamical parameters have the constraint $(a+d)=0.45$, the values of $a$ fall in the range $0.38-0.45$ so that the arguments $k a$ of $Z_{n}$ are between 8 and 126. The evaluation of $Z_{n}$ in $k d$ requires more attention as $d$ becomes small, because there is a cutoff on the order of $J_{n}$, and $Y_{n}$ may diverge. We have used the IMSL routines to evaluate the $Z_{n}$ and, for the superior limit $(k b) \approx 278$, we have 15 siginificant digits converged for $n=0-847$. To have the same precision for the inferior limit $(k d) \rightarrow 0$, we have gone the closest possible to the concentric case, but this has varied depending on the local density of levels and mainly if there was some "fast" level near the doublet under observation. For instance, when $\arg =10^{-6}$ we evaluate $Z_{n}$ with $n$ up to 41 , for $\arg =10^{-3}$ the limiting $n$ is 61 , and when $\arg =10^{-2} n$ ends in 81. Even though difficulties may occur when $d \rightarrow 0$, we point out that the more integrable the system is, the more stable the doublets; hence, it is possible to follow them in a safe way. With these cautions, we construct the matrices and next we evaluate its determinant also through IMSL. The determinant is a function of $k$, $\operatorname{det}(k)$, and it may become null if we find a root or if all elements of a line, or of a column, are nulls. This latter possibility occurs with relative frequency and it constitutes a natural condition to truncate the determinant. From the numerical point of view, we stipulate a precision $\epsilon \approx 10^{-15}$ and we consider the matrix elements non-null when their absolute values are greater than eps. From there we look for the maximum order of the determinant. The roots of $\operatorname{det}(k)$ are searched by the bisection method and the precision of the eigenvalues is verified by varying the order of the determinant. As we increase or decrease the order, we observe how many digits have converged and we compare this with the local mean spacing of levels. Firstly, we compute the Weyl density of levels [17] for this billiard $\left[\bar{N}(k)=(1 / 4 \pi)\left(A k^{2}\right.\right.$ $-L k)]$ in a very small window of $k$ and the mean spacing in this window, for a small range of $d$, where $A$ is the area of the ring and $L$ is the perimeter along the borders. Next, we ask if the of the eigenvalues is greater than the mean spacing. If not, we neglect the eigenvalues and go on; otherwise, we keep them. Finally, the numerical results concerning the 
a)

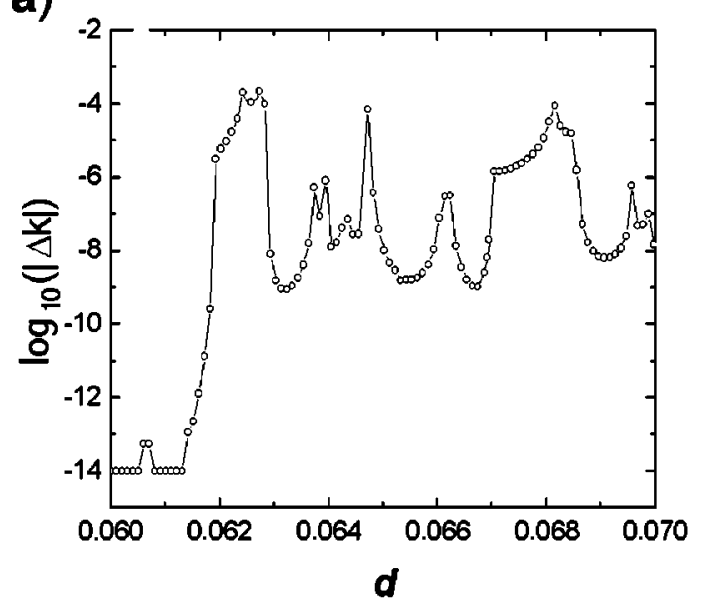

c)

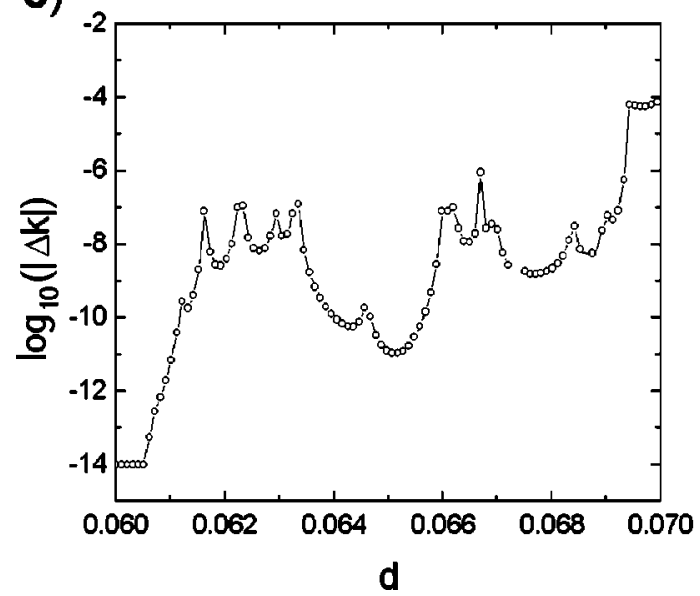

b)

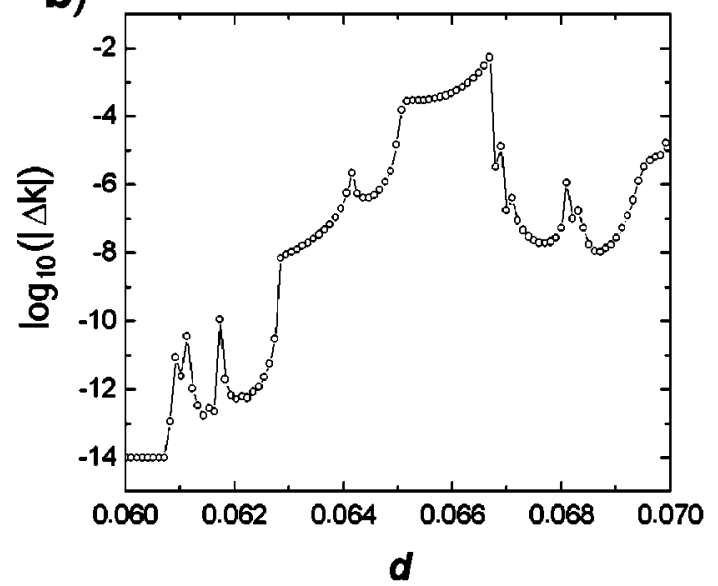

d)

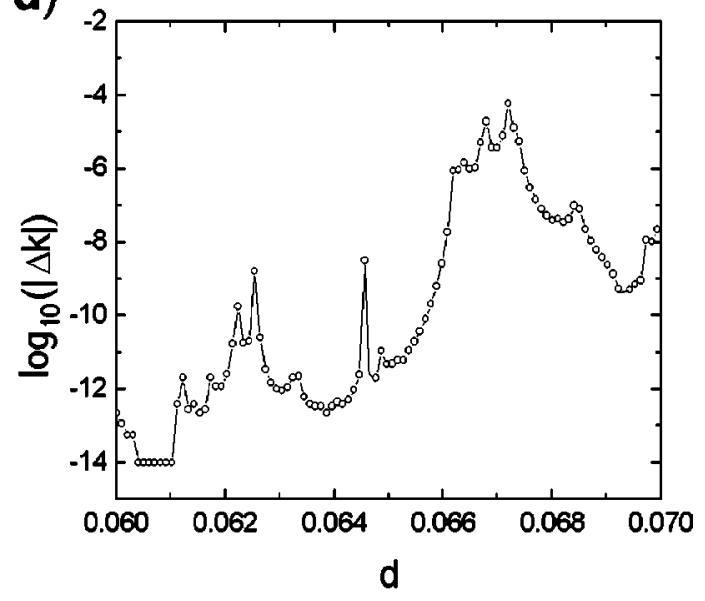

FIG. 3. Dynamics of $\log _{10}|\Delta k|$ versus $d$ exhibiting the fluctuations for the semiclassical doublets listed in Table I. Observe that they are very close in phase space but their behaviors are very different.

splitting dynamics are presented in Figs. 3-7.

\section{DOUBLET DISTRIBUTION}

In order to analyze the statistical effects of dynamical tunneling, we first calculate the unperturbed eigenwavenum-

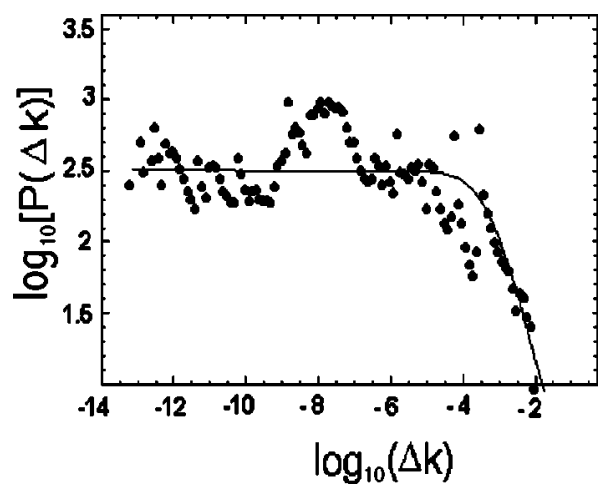

FIG. 4. Splitting fluctuations distribution using the four semiclassical doublets. The points are our numerical results, while the full line represents the analytical results by Leyvraz and Ullmo. bers and their position on phase space. In Table I, we present four of them. We have fixed the quantum number $n$ to define a family having the same angular momentum, and we chose four consecutive (in the quantum number $m$ ) eigenwavenumbers near the caustic $S=0.45$ to amplify the coupling between chaotic states and doublet states, when $d \neq 0$. The family chosen, $n=125$, corresponds to an extremely high quantum number, and the associated eigenwavenumbers are also in a scale of very high energies. In comparison, the unperturbed fundamental state has $k \approx 5.66$ and the value of the doublet \#4 of Table I, $k \approx 277$, would closely correspond to the 7400th level if the whole spectrum had been obtained. For this reason, we call this family, $n=125$, semiclassical doublets.

In a second moment, we dislocate the inner circle up $d$ $\approx 0.06$, which is sufficient to initialize classical transport between the upper and the lower WGO regions, and we search for the solutions of Eq. (3) in a small range of values of $d$ $=0.06-0.07$. In Fig. 3 we exhibit the behavior of the splittings of the selected doublets, where we see a lot of fluctuations even with nearly unmodified classical dynamics. The splittings have varied many orders of magnitude, and the fluctuations are due to the high density of levels in the semiclassical regime, which leads to several quasicollisions 


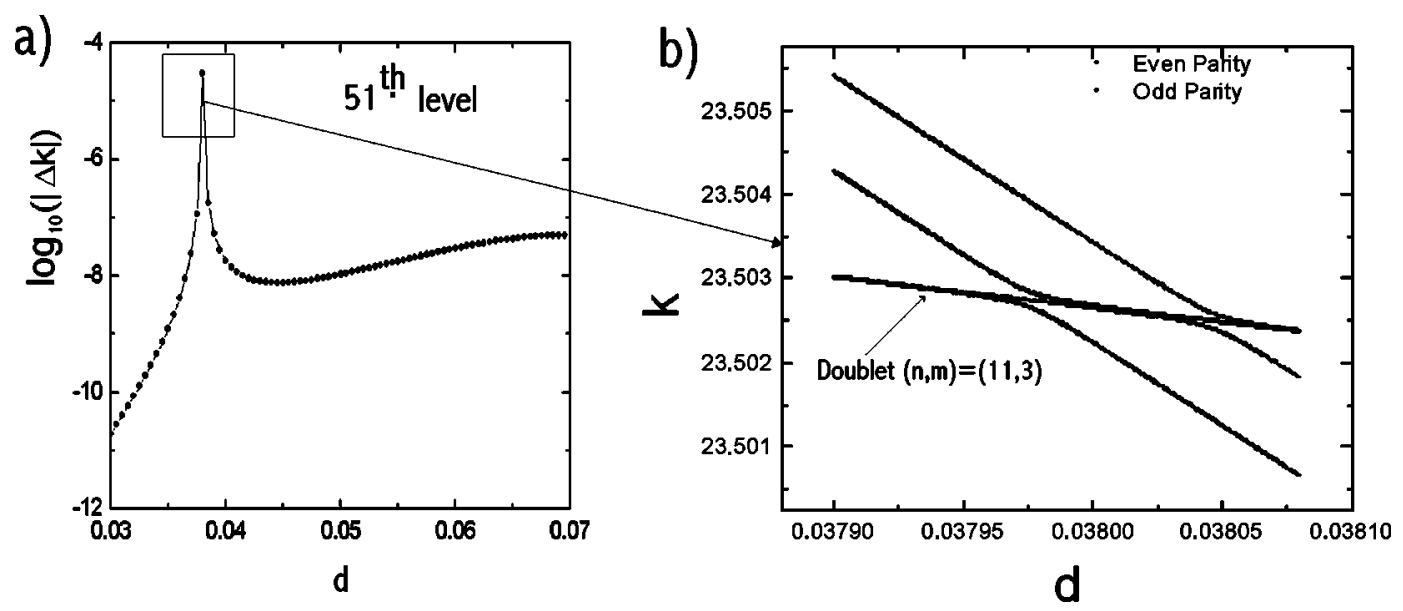

FIG. 5. (a) A single peak in the dynamics of $\log _{10}|\Delta k|$ versus $d$ for the quantum doublet of Table II. (b) Enlargement around the peak showing two avoided crossings involving a doublet and two singlets.

among chaotic (or even regular) levels with the doublets. We will return to this point later. For the same reason, the calculations needed a high numerical precision. Although the doublets are neighbors in phase space and pertaining to the same family $n=125$ (see Table I), we found different behaviors for their quantum dynamical splitting. The origins of these differences are not clear as yet. The occurrence of these fluctuations makes the chaotic tunneling denomination, introduced by Mouchet and Delande [6], more adjusted to denote the influence of classical chaos on the dynamical tunneling process.

Since the dynamics of the doublet splittings is established, we construct an ensemble of splittings in the cited range of $d$ and with the chosen semiclassical family, $n=125$. The interval of $d$ is divided into 500 subintervals, and we calculate the splittings of the four selected doublets for each value of $d$ in such way the ensemble has 2000 components. For each value of splitting, we ask how many splittings have fallen inside it and we plot the probability of each one occurring in a scenario wherein chaotic tunneling is the dominant mechanism and the classical dynamics is near constant. In Fig. 4, we present a $\log _{10}-\log _{10}$ plot of this probability, which shows that the analytical predictions (see Ref. [11] for details) agree in a very good way with the average behavior of the numerical results even though some deviations exist. This point is still not clear because in this semiclassical regime there are many near collisions and consequently many fluctuations due

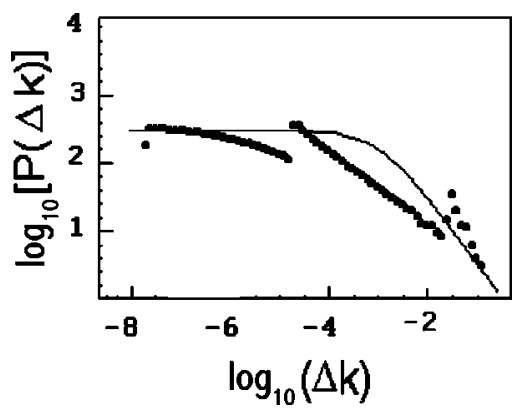

FIG. 6. Splitting fluctuation distribution using the quantum doublet. to the high density of levels, so that we would expect that a certain splitting was nearly equally probable to occur, in a range of splittings before the crossover point, and for the same reason the probability should decay nearly uniformly. We suppose that these deviations may be related to the different behavior cited above concerning the splittings presented in Fig. 3.

In order to verify the extension of the applicability of the theoretical results presented in Ref. [11], we prepare another ensemble, but now in a different regime. We choose a doublet near the botton of the spectrum with low quantum number $n=11$, low eigenwavenumber $k \approx 23.75$ (for $d=0$ ), and also neighbor to the caustic, as presented in Table II. If we had obtained the whole spectrum this value of $k$ would correspond to the 51st level. We call this doublet a quantum doublet.

In this scale of the spectrum, the mean spacing of levels is greater than the one in the semiclassical regime, or in other words, the spectrum has another $\hbar$ scale. An immediate consequence is the decreasing of quasicollisions between other levels and the doublet, and thus there is a reduction of the fluctuations, as may be observed in Fig. 5. In Fig. 5(a), we

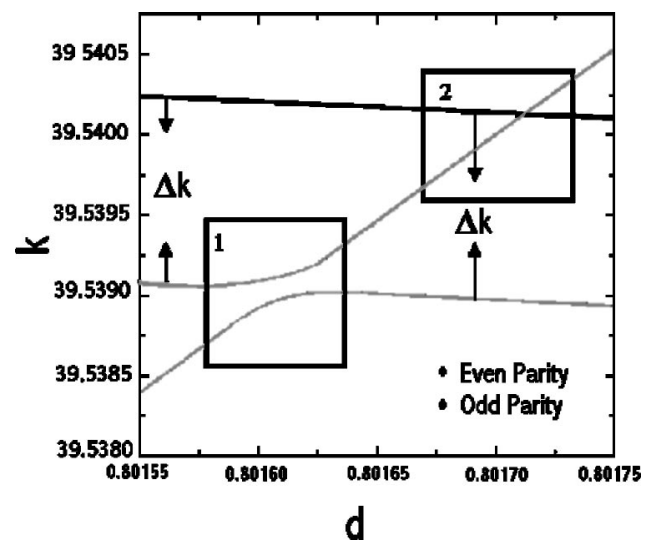

FIG. 7. A singlet-doublet interaction. An avoided crossing between levels of same parity, a crossing between levels of distinct parities, and the nondegenerate doublet during the entire interaction are shown. This doublet is a new one, not listed in the Tables. 
TABLE I. Four semiclassical doublets. They are identified by the quantum numbers $(n, m)$ and belong to the family $n=125$. $S$ identifies their position on phase space; they are near the caustic. The $k_{n, m}$ are the eigenwavenumbers when $d=0$.

\begin{tabular}{ccccc}
\hline \hline Doublet & $n$ & $m$ & $k_{n, m}$ & $S=n / k$ \\
\hline 1 & 125 & 31 & 266.22885057777 & $4.6952086420657 \times 10^{-1}$ \\
2 & 125 & 32 & 269.86711513899 & $4.6319092986049 \times 10^{-1}$ \\
3 & 125 & 33 & 273.56230674740 & $4.5693429583271 \times 10^{-1}$ \\
4 & 125 & 34 & 277.33406694187 & $4.5071996159129 \times 10^{-1}$ \\
\hline \hline
\end{tabular}

present the dynamics of the doublet splitting as function of $d$ where a single peak is visible. In Fig. 5(b), we plot values of $k$ around the peak, where the $(n, m)=(11,3)$ near-degenerate doublet appears together with two singlets of different parities. There are two avoided crossings that lead in fact to two peaks, but the second is not visible using the scale of Fig. 5(a).

An effect of the $\hbar$ scale is that the doublet acquires a significant spacing even when $d=0$. We construct the ensemble with the chosen doublet in the range $d=0.03-0.04$ (see Fig. 2) divided into 2000 subintervals. In this context, chaos does not connect the WGO regions; thus, there is no dynamical tunneling effect. In Fig. 6, we present the numerical splitting distribution (dots) for this quantum doublet together with the corresponding analytical formula (full line) from Ref. [11]. We note that the analytical prediction again fits the numerical results in a reasonable way. A similar result, in a different system, has been noticed by Mouchet and Delande [6]. In both works, the systems are quasi-integrable, thus, dynamical tunneling is not the dominant mechanism, or it does not exist.

\section{COLLISION BETWEEN A SINGLET AND A DOUBLET}

As we have already pointed out, the near collisions of levels with a doublet induce fluctuations on the doublet splitting. For instance, if a level of a specific parity goes towards the doublet, there are two possibilities: it will find (i) the doublet level of different parity or (ii) the doublet level of same parity.

In the first scenario, the uncorrelated doublet level follows its trajectory while the others of same symmetry repel themselves, increasing the splitting and producing peaks as seen in Figs. 3 and 5(a). As an example we present, in Fig. 5(b), two near collisions of two singlets of different parities with the doublet $(n, m)=(11,3)$ at two values of the pertubation parameter. They generate two closely spaced peaks in the rectangle seen in Fig. 5(a), which may be explained through this first scenario.
In the second scenario, they will repel each other, hurling the level doublet towards its partner of the other parity, increasing the degeneracy [see Ref. [6]]. Even though it is theoretically possible to obtain an exact degeneracy between partners of a doublet, it is worthwhile to emphasize that up this stage we did not find it in our numerical calculations. Actually, we have found another situation for the second scenario, not mentioned by the authors of Ref. [6], which does not include the appearing of crossing of levels of a doublet. This is shown in Fig. 7. It is important to point out that in this case the doublet does not correspond to the ones listed in the tables; we have sought it in a convenient range of $k$ and of $d$ (see the figure scale). In this numerical example, a singlet of odd parity comes from the lower part of the plot towards the doublet. In this three-level context, the two levels of same parity are consecutive in the spectrum. The repulsion between them (rectangle 1) makes the old odd doublet level cross the even doublet level (rectangle 2). However, looking at the crossing point in the plot, we perceive that the doublet is well defined by two parallel straight lines and hence there is no doublet degeneracy. The splittings, before and after the avoided crossing, are indicated by arrows and $\Delta k$. What happened is as usual, at an avoided crossing, the involved levels exchange their roles in the level dynamics.

\section{CONCLUSIONS}

We have chosen a very convenient model, the annular billiard, to study numerically the splittings distribution in the semiclassical and quantum regimes as well as the local quantum dynamics of three levels. This model preserves a discrete symmetry even when there is chaos in the classical dynamics, and due to that, dynamical tunneling may occur in the quantum counterpart. This latter is strongly influenced by chaos, but due to the several near collisions occurring in the quantum dynamics, there are fluctuations in the splitting magnitudes. These fluctuations were evaluated with good quantum numbers, or families of the same angular momentum. In the semiclassical regime, we have taken a set of

TABLE II. A quantum doublet near the botton of the spectrum belonging to the family $n=11$. It is near the caustic.

\begin{tabular}{ccccc}
\hline \hline Doublet & $n$ & $m$ & $k_{n, m}$ & $S=n / k$ \\
\hline 5 & 11 & 3 & 23.747794041989 & $4.6320091796949 \times 10^{-1}$ \\
\hline \hline
\end{tabular}


extremely high doublets in a context where chaotic tunneling is the dominant mechanism, and we have verified that analytical predictions [11] are in good agreement with the mean behavior of our numerical results. The observed deviations should be investigated in more detail in order to clarify what is their origin. This agreement has also been observed with a low doublet where there is no chaotic tunneling. It seems for us that chaotic tunneling is only sufficient, but not necessary, for the applicability of the Leyvraz and Ullmo distribution.

We have also presented a numerical example concerning the local process of collision involving a singlet with a doublet. In our investigations up to now, no exact doublet degeneracy was observed, while the peaks were well described through the correlation between the level of the doublet and the singlet. These points will be analyzed in future calcula- tions. To finish, we would like to emphasize that all computational calculations to follow a level or a doublet were done since the near-concentric configuration, $d \approx 0$, especially for the semiclassical case because there are too many closely spaced levels in the spectrum. Moreover, the increment of $d$ was as small as possible. With these numerical contributions, we believe that a little more is understood concerning quantum chaos.

\section{ACKNOWLEDGMENTS}

We would like to thank the Brazilian agency Fapesp for partial financial support through the process 01/11840-2. We also thank A. Mouchet and J.S.E. Ortiz for useful discussions.
[1] M.J. Davis and E.J. Heller, J. Phys. Chem. 75, 246 (1981).

[2] R. Egydio de Carvalho, Nonlinearity 6, 973 (1993).

[3] E.J. Heller, J. Phys. Chem. A 103, 10433 (1999).

[4] W.A. Lin, and L.E. Ballentine, Phys. Rev. A 45, 3637 (1992).

[5] O. Bohigas, S. Tomsovic, and D. Ullmo, Phys. Rep. 223, 43 (1993).

[6] A. Mouchet and D. Delande, Phys. Rev. E 67, 046216-1 (2003); for a complementary reading, see A. Mouchet, C. Miniatura, R. Kaiser, B. Grémaud, and D. Delande, Phys. Rev. E 64, 016221 (2001).

[7] K. Takatsuka and H. Ushiyama, Phys. Rev. A 51, 4353 (1995); see also Phys. Rev. E 53, 115 (1996).

[8] S. Takada, P.N. Walker, and M. Wilkinson, Phys. Rev. A 52, 3546 (1995).

[9] S.D. Frischat and E. Doron, Phys. Rev. E 57, 1421 (1998); E. Doron, and S.D. Frischat, Phys. Rev. Lett. 75, 3661 (1995).
[10] For an overview about random matrices see, O. Bohigas and M.J. Giannoni, Lecture Notes in Physics (Springer, Berlin, 1984), Vol. 209; T. Guhr, A.M. Groeling, and H.A. Weidenmüller, Phys. Rep. 299, 189 (1998).

[11] F. Leyvraz and D. Ullmo, J. Phys. A 29, 2529 (1996).

[12] O. Bohigas, D. Boosé, R. Egydio de Carvalho, and V. Marvulle, Nucl. Phys. A 560, 197 (1993).

[13] N. Saitô, H. Hirooka, J. Ford, F. Vivaldi, and G.H. Walker, Physica D 5, 273 (1982).

[14] G.S. Singh and L.S. Kothari, J. Math. Phys. 25, 810 (1984).

[15] K. Nagaya, J. Sound Vib. 50, 545 (1977).

[16] J.S. Espinoza Ortiz and R. Egydio de Carvalho, Braz. J. Phys. 31, 538 (2001).

[17] H.P. Baltes and E.R. Hilf, Spectra of Finite Systems (Bibliographisches Institut, Mannheim, 1976). 